Linköping Studies in Science and Technology.

Dissertations, No. 1886

\title{
Error analysis of summation-by-parts formulations: Dispersion, transmission and accuracy
}

\author{
Viktor Linders
}

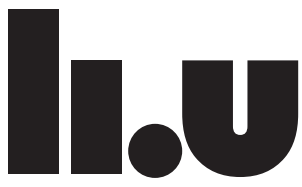

LINKÖPING UNIVERSITY

Department of Mathematics, Division of Computational Mathematics Linköping University, SE-581 83 Linköping, Sweden

Linköping 2017 
Linköping Studies in Science and Technology. Dissertations, No. 1886

Error analysis of summation-by-parts formulations: Dispersion, transmission and accuracy

Copyright (C) Viktor Linders, 2017

Division of Computational Mathematics

Department of Mathematics

Linköping University

SE-581 83, Linköping, Sweden

viktor.linders@liu.se

www.liu.se/mai/ms

Typeset by the author in $\mathrm{AT}_{\mathrm{E}} \mathrm{X} 2 \mathrm{e}$ documentation system.

ISSN 0345-7524

ISBN 978-91-7685-427-3

Printed by LiU-Tryck, Linköping, Sweden 2017 
To my family. 



\section{Abstract}

In this thesis we consider errors arising from finite difference operators on summation-by-parts (SBP) form, used in the discretisation of partial differential equations. The SBP operators are augmented with simultaneous-approximationterms (SATs) to weakly impose boundary conditions. The SBP-SAT framework combines high order of accuracy with a systematic construction of provably stable boundary procedures, which renders it suitable for a wide range of problems.

The first part of the thesis treats wave propagation problems discretised using SBP operators on coarse grids. Unless special care is taken, inaccurate approximations of the underlying dispersion relation materialises in the form of an incorrect propagation speed. We present a procedure for constructing SBP operators with minimal dispersion error. Experiments indicate that they outperform higher order non-optimal SBP operators for flow problems involving high frequencies and long simulation times.

In the second part of the thesis, the formal order of accuracy of SBP operators near boundaries is analysed. We prove that the order in the interior of a diagonal norm based SBP operator must be at least twice that of the boundary stencil, irrespective of the grid point distribution near the boundary. This generalises the classical theory posed on uniform and conforming grids. We further show that for a common class of SBP operators, the diagonal norm defines a quadrature rule of the same order as the interior stencil. Again, this result is independent of the grid.

In the final contribution if the thesis, we introduce the notion of a transmission problem to describe a general class of problems where different dynamics are coupled in time. Well-posedness and stability analyses are performed for continuous and discrete problems. A general condition is obtained that is necessary and sufficient for the transmission problem to satisfy an energy estimate. The theory provides insights into the coupling of fluid flow models, multi-block formulations, numerical filters, interpolation and multi-grid implementations. 



\section{Sammanfattning på svenska}

Partiella differentialekvationer ligger till grund för matematiska modeller av många ämnesspecifika problem inom t.ex. fysik, kemi, ekonomi och meteorologi. Vanligen angrips sådana problem numeriskt genom att ersätta de styrande ekvationerna med en lämplig diskret approximation, vilken i sin tur kan lösas med hjälp av en dator. Det är emellertid oundvikligt att den numeriska proceduren introducerar fel i lösningen. I allmänhet är det utmanande att analysera dessa fel, samt att både begränsa och minimera dem. I denna avhandling beaktas just sådana utmaningar.

En method som begränsar tillväxten av numeriska fel sägs vara stabil. För finita differensapproximationer på partiell summationsform (eng. "summationby-parts", SBP) kan stabilitet systematiskt visas om rand- och begynnelsevillkor implementeras på svag form. Att minimera felen är emellertid ett separat problem och beror på de styrande ekvationernas natur.

Avhandlingen inleds med en analys av lågupplösta SBP-approximationer av ekvationer vars lösningar antar en vågform. För högfrekventa vågor uppstår så kallade dispersionsfel, vilka tar sig uttryck i felaktiga våghastigheter. I avhandlingen utvecklas en metod för att konstruera nya SBP-approximationer med minskat dispersionsfel så att detta fenomen minimeras.

I avhandlingens andra del studeras SBP-approximationers noggrannhetsordning, dvs. den takt med vilken det numeriska felet minskar då approximationens upplösning förfinas. Här presenteras ett bevis för att noggrannhetsordningen vid beräkningsdomänens gränser som mest är hälften av ordningen i domänens inre. Detta resultat gäller även om approximationen är finare upplöst vid ränderna än i domänens övriga delar.

Slutligen behandlar avhandligen så kallade transmissionsproblem, vilka beskriver scenarier då olika dynamiska system sammankopplas i tiden. Både differentialekvationer och diskreta approximationer analyseras och ett allmänt stabilitetsvillkor härleds. Transmissionsproblem är vanligt förekommande, både som ekvationer och i samband med deras diskretiseringar. Den härledda teorin för dessa problem ger således insikter i fysikaliska fenomen såväl som en bred arsenal numeriska tekniker, exempelvis filtrering och interpolation. 



\section{List of Papers}

This thesis is based on the following papers, which henceforth will be referred to by their roman numerals:

I. Linders, V. \& Nordström, J. (2015). Uniformly best wavenumber approximations by spatial central difference operators. Journal of Computational Physics, 300, 695-709.

II. Delorme, Y. T., Puri, K., Nordström, J., Linders, V., Dong, S. \& Frankel, S. H. (2017). A simple and efficient incompressible Navier-Stokes solver for unsteady complex geometry flows on truncated domains. Computers \& Fluids, 150, 84-94.

III. Linders, V., Kupiainen, M. \& Nordström, J. (2017). Summation-by-Parts operators with minimal dispersion error for coarse grid flow calculations. Journal of Computational Physics, 340, 160-176.

IV. Linders, V., Lundquist, T. \& Nordström, J. (2017). On the order of accuracy of finite difference operators on diagonal norm based summationby-parts form. Linköping University Press, LiTH-MAT-R-2017/11-SE (Submitted).

V. Nordström, J. \& Linders, V. (2017). Well-posed and stable transmission problems. Linköping University Press, LiTH-MAT-R-2017/15-SE (Submitted).

With the exception of paper II, I have contributed to the above listed papers by deriving most of the novel theoretical results as well as performing the numerical calculations. I have written the manuscripts myself with editorial support from Jan Nordström, Marco Kupiainen and Tomas Lundquist. My contribution to paper II includes deriving and providing the difference stencils used to obtain the numerical results as well as selecting appropriate numerical filters to match the stencils. 



\section{Acknowledgements}

It goes without saying that the content of this thesis is the result of the efforts and engagements of many people. The list is longer than I have made space for, though I suspect that they know who they are.

First, I would like to express my gratitude to my advisor, Jan Nordtröm, without whom this venture would not have commenced, continued nor concluded. His determination, perseverance and patience has been key to any success I have enjoyed during the past few years. I must also thank my second advisor, Marco Kupiainen, for much the same reasons. He has taught me everything I know about fast computers, small planes, huge ships and cramped caves.

I am sincerely grateful to the many friends and colleagues that I have been fortunate enough to get to know; some through work, others through $\mathrm{PhD}$ student commitments, but most through the efforts you have made to turn Linköping into the wonderful place it is. This acknowledgement is incomplete until I have raised my glass to chant for the Kören!

Among those who have made especially important contributions to this thesis, I must mention Tomas Lundquist. His devotion to understanding is unprecedented and has been a great inspiration.

I must also give my thanks to Cristina La Cognata. Simply put, she made me better; as a researcher and as a person.

My family deserves all the gratitude I can muster, but I suppose they already know that. This thesis is dedicated to them.

Last, but certainly not least, to my love and best friend, Anna. In some sense you are the antipole of this thesis; void of errors, yet of unbounded energy.

Viktor Linders

Linköping, 2017 



\section{Contents}

Abstract

Sammanfattning på svenska v v

List of Papers vii

Acknowledgements $\quad$ ix

1 Introduction 1

2 Well-posedness and stability 3

2.1 Well-posed problems ................ 3

2.2 Stable discretisations . . . . . . . . . . . . . . . . 4

$\begin{array}{lll}3 & \text { The energy method } & 7\end{array}$

3.1 Strong boundary conditions . . . . . . . . . . . . . 7

3.2 Weak formulations . . . . . . . . . . . . . . 8

3.3 Transmission ................... 8

4 Summation-by-parts $\quad 11$

4.1 Semi-discretisations . . . . . . . . . . . . . . . 11

4.2 SBP in time . . . . . . . . . . . . . . . 12

4.3 Second derivatives . . . . . . . . . . . . . . . . 13

4.4 Generalised SBP . . . . . . . . . . . . . . . . . . . 14

5 Finite difference operators $\quad \mathbf{1 5}$

5.1 The structure of $P$ and $Q \ldots \ldots \ldots$. . . . . . . . 15

5.2 Accuracy and convergence . . . . . . . . . . . 16

6 Dispersion $\quad 19$

6.1 Analytic and numeric dispersion relations . . . . . . . . . . . . 19

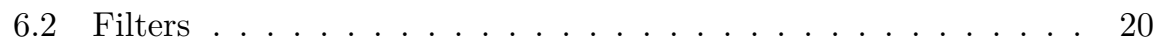

$\begin{array}{lll}7 & \text { Summary of papers } & 23\end{array}$ 
References $\quad \mathbf{2 6}$

I. Uniformly best wavenumber approximations by spatial central difference operators . . . . . . . . . . . . . . . . . 29

II. A simple and efficient incompressible Navier-Stokes solver for unsteady complex geometry flows on truncated domains . . . . . . 47

III. Summation-by-parts operators with minimal dispersion error for coarse grid flow calculations . . . . . . . . . . . . . 61

IV. On the order of accuracy of finite difference operators on diagonal norm based summation-by-parts form . . . . . . . . . . 81

V. Well-posed and stable transmission problems _. . . . . . . . . . 99 


\section{Introduction}

Partial differential equations (PDEs) form a fundamental tool in the mathematical modelling of a plethora of natural phenomena arising in a variety of scientific disciplines. Important examples include fluid flow, electromagnetic interaction and wave motion. For typical realistic models, analytic solutions are beyond reach. Instead, one resorts to numerical methods, by approximating the governing equations at a discrete set of grid points. Such procedures are accompanied both by theoretical difficulties and computational challenges. Nonetheless, the development of computer technology during the past decades has spurred a spiralling demand for increasingly complex models to be attacked by numerical means. Consequently, the need of efficient and robust numerical methods has been steadily increasing.

A fundamental question pertaining to any PDE is whether or not it is well-posed, i.e. if a unique solution exist that depends continuously on available data. A well behaved model must answer this question in the affirmative. Similarly, corresponding numerical approximations should stand the test of stability; the discretisations must not support unphysical growth of the solution. The latter is generally difficult to achieve close to the boundaries of the computational domain, in particular for methods with high order of accuracy. Yet, high order is desirable for an efficient implementation.

Summation-by-parts (SBP) operators, augmented with simultaneous approximation terms (SAT) for weak boundary treatments [4], offer a systematic approach towards provably stable, yet high order accurate numerical approximations. By mimicking certain properties of the underlying PDEs, an SBP-SAT discretisation yields a numerical solution that satisfies bounds analogous to the true solution of the well-posed governing equations. Although originally proposed for finite difference methods [9], the SBP framework has been extended to methods outside this paradigm, including finite volume methods [12, 13], spectral Galerkin and element methods $[3,6]$, correction procedures via reconstruction [15] and temporal discretisations [14].

In any numerical approximation, errors are inevitably present. In this thesis, we are primarily concerned with the errors of finite difference stencils on SBP form. The first part of the thesis develops a theory of dispersion errors for finite difference approximations of wave propagation problems. Such errors materialise in the form of incorrect propagation speeds, which has a pronounced 
impact on the quality of numerical solutions on coarse grids. New SBP operators are developed that minimise the dispersion errors in a certain sense. Numerical simulations suggest that these optimised operators perform well for problems posed on grids that are too coarse for standard techniques.

Of further interest is the order of accuracy of an SBP operator, which reveals the asymptotic behaviour of the error as the grid is progressively refined. A classic theorem states that on a uniform grid, the accuracy near domain boundaries is at most half that of the interior domain [9]. The second contribution of the thesis is the generalisation of this result to arbitrary grids, which may or may not coincide with the physical domain boundaries. Further, it is shown that the accuracy of the quadrature rule (or mass matrix), inherent in the definition of the SBP operator, is limited by the accuracy of the operator in the interior domain.

Practical implementations of numerical methods certainly encompass more than just discretising the PDE. Typically, various techniques are employed in order to reduce the errors or improve the efficiency of the numerical method at hand. Examples include interpolating multi-block techniques, multi-grid accelerators and numerical filters. For each technique, it must be guaranteed that the errors introduced are bounded such that the resulting implementation remains stable. The final part of the thesis treats a general class of problems, referred to as transmission problems, for which the above listed techniques arise as special cases. Conditions for well-posedness and stability are derived through energy estimates and bounds on the solutions are obtained via scaling arguments.

The introductory chapters of this thesis are organised as follows: In Chapter 2 , the notions of well-posedness and stability are formalised. We continue by introducing the energy method in Chapter 3 and apply it to a model problem. The SBP-SAT framework is introduced in Chapter 4 and its efficacy for obtaining stable discretisations is demonstrated using the discrete energy method. Specific details about the structure and properties of finite difference stencils on SBP form are presented in Chapter 5. In Chapter 6, a brief theory of dispersion errors of finite difference stencils is introduced, together with a discussion of numerical filters. The papers constituting the major part of the thesis are briefly summarised in Chapter 7. We remark that for consistency of the introductory chapters, some notational differences may occur relative to the subsequent papers. 


\section{Well-posedness and stability}

As indicated in the previous chapter, the concepts of well-posedness and stability are fundamental in the theory of PDEs and their numerical approximations. In the following, we will formalise these notions. We further introduce the concept of a semi-bounded operator. In due course we will see that constructing semi-bounded derivative approximations provides a systematic way of ensuring stability of numerical schemes.

\subsection{Well-posed problems}

Consider the linear initial-boundary value problem (IBVP)

$$
\begin{aligned}
& u_{t}+\mathcal{D}(u)=F, \quad t>0, \quad x \in \Omega, \\
& \mathcal{B}(u)=g, \quad t \geq 0, \quad x \in \Gamma, \\
& u=f, \quad t=0, \quad x \in \Omega \cup \Gamma,
\end{aligned}
$$

where $\Omega$ is an open $d$-dimensional region and $\mathcal{D}$ is a differential operator with smooth coefficients. The operator $\mathcal{B}$ defines a set of boundary conditions on the boundary $\Gamma$ of $\Omega$. The functions $F, g$ and $f$ are given forcing, boundary and initial data, which we assume are smooth and compatible.

For two functions $u$ and $v$ defined on $\Omega$, we introduce the inner product and norm

$$
(u, v)_{H}=\int_{\Omega} u^{\top} H v \mathrm{~d} x, \quad\|u\|_{H}=(u, u)_{H}^{1 / 2},
$$

where $H$ is a positive definite matrix.

Definition 2.1.1. Let $\mathbb{V}$ be the space of differentiable functions satisfying the boundary conditions $\mathcal{B}(v)=0$. The differential operator $\mathcal{D}$ is semi-bounded if for all $v \in \mathbb{V}, \mathcal{D}(v)$ satisfies the inequality

$$
(v, \mathcal{D}(v))_{H} \geq-\alpha\|v\|_{H}^{2},
$$

for some real constant $\alpha$ independent of of $v$. It is maximally semi-bounded if it is semi-bounded in the space $\mathbb{V}$ but not in any space with fewer boundary conditions. 
Note that if $\mathcal{D}$ is semi-bounded in $(2.1)$, and $F=g=0$, an estimate of the solution $u$ is viable by considering

$$
\frac{\mathrm{d}}{\mathrm{d} t}\|u\|_{H}^{2}=2\left(u, u_{t}\right)_{H}=-2(u, \mathcal{D}(u))_{H} \leq 2 \alpha\|u\|_{H}^{2},
$$

which after integration gives

$$
\|u(x, t)\|_{H}^{2} \leq e^{2 \alpha t}\|f(x)\|_{H}^{2} .
$$

Definition 2.1.2. The IBVP (2.1) is well-posed if, for sufficiently smooth and compatible data, it has a unique smooth solution satisfying the estimate

$$
\|u\|_{I}^{2} \leq K(t)\|f\|_{I I}^{2}
$$

where $K(t)$ is independent of $u$ and the data. It is strongly well-posed if it satisfies the estimate

$$
\|u\|_{I}^{2} \leq K(t)\left(\|f\|_{I I}^{2}+\|F\|_{I I I}^{2}+\|g\|_{I V}^{2}\right) .
$$

The norms involved in (2.5) and (2.6) are generally different. Comparing (2.4) and (2.5), it is clear that if a unique solution exists, semi-boundedness of $\mathcal{D}$ imples well-posedness of (2.1). For linear problems, maximal semi-boundedness imples existence and uniqueness, and is thus sufficient for well-posedness. We say that the solution $u$ satisfies an energy estimate if it is bounded in terms of data such as in (2.5) or (2.6).

It follows from (2.6) that the solution of a well-posed problem depends continuously on the available data. Consider (2.1) with perturbed data $F+\delta F, g+\delta g$ and $f+\delta f$, and solution $v$. Then, if the problem is well-posed, the difference $w=v-u$ satisfies the estimate

$$
\|w\|_{I}^{2} \leq K(t)\left(\|\delta f\|_{I I}^{2}+\|\delta F\|_{I I I}^{2}+\|\delta g\|_{I V}^{2}\right) .
$$

Thus, small perturbations in the data correspond to small variations in the solution.

\subsection{Stable discretisations}

Stability may be viewed as the discrete analogue of well-posedness. Consider the semi-discretisation of (2.1) given by

$$
\begin{aligned}
\mathbf{u}_{t}+D(\mathbf{u}, \mathbf{g}) & =\mathbf{F}, \quad t>0 \\
\mathbf{u} & =\mathbf{f}, \quad t=0
\end{aligned}
$$

where $\mathbf{u}^{\top}=\left(\mathbf{u}_{1}^{\top}, \ldots, \mathbf{u}_{d}^{\top}\right)$ with $\mathbf{u}_{j}^{\top}=\left(u_{0}^{(j)}, \ldots, u_{n_{j}}^{(j)}\right), j=1, \ldots, d$ is a grid vector approximating the function $u(x, t)$ on the $d$-dimensional grid $\mathbf{x}^{\top}=\left(\mathbf{x}_{1}^{\top}, \ldots, \mathbf{x}_{d}^{\top}\right)$. Here, $\mathbf{x}_{j}^{\top}=\left(x_{0}^{(j)}, \ldots, x_{n_{j}}^{(j)}\right)$ and $x_{k}^{(j)}=x_{0}^{(j)}+\sum_{l=1}^{k} h_{l}^{(j)}$, where $h_{l}^{(j)}$ denotes local step sizes in the $j$ th spatial dimension. 
The spatial discretisation $D(\mathbf{u}, \mathbf{g})$ approximates the differential operator $\mathcal{D}$ augmented with the boundary operator $\mathcal{B}$. The grid vectors $\mathbf{f}, \mathbf{g}$ and $\mathbf{F}$ are obtained by projecting the data $f, g$ and $F$ onto the spatial grid.

We introduce the discrete inner product and norm

$$
(\mathbf{u}, \mathbf{v})_{h}=\mathbf{u}^{\top} P \mathbf{v}, \quad\|\mathbf{u}\|_{h}=(\mathbf{u}, \mathbf{u})_{h}^{1 / 2},
$$

where the positive definite matrix $P$ is such that (2.8) approximates the continuous inner product and norm in (2.2) on the grid $\mathbf{x}$.

Definition 2.2.1. The semi-discretisation (2.7) is stable if, for sufficiently smooth and compatible data and sufficiently fine grids, it has a unique and smooth solution satisfying the estimate

$$
\|\mathbf{u}\|_{I_{h}}^{2} \leq K_{d}(t)\|\mathbf{f}\|_{I I_{h}}^{2},
$$

where $K_{d}$ is independent of $\mathbf{u}$, the grid and the data. It is strongly stable if it satisfies the estimate

$$
\|\mathbf{u}\|_{I_{h}}^{2} \leq K_{d}(t)\left(\|\mathbf{f}\|_{I I_{h}}^{2}+\|\mathbf{F}\|_{I I_{h}}^{2}+\|\mathbf{g}\|_{I I I_{h}}^{2}\right) .
$$

Here, 'smooth data' refers to the projection of a smooth function onto the grid. Note that if $\mathbf{F}=\mathbf{g}=\mathbf{0}$ and $D(\mathbf{u}, \mathbf{0})$ in $(2.7)$ is semi-bounded in the inner product (2.8), an energy estimate follows in the same way as for the semibounded continuous problem.

Much like for the continuous problem, the energy estimate (2.9) implies that small perturbations in the data correspond to small variations in the semidiscrete solution. This is of particular importance when the perturbations are representations of truncation errors in the numerical approximation. In this case, (2.9) guarantees that the errors inherent to a stable discretisation will not be uncontrollably amplified.

Of course we must also discretise time prior to implementation, whence we find ourselves with a fully discrete problem. We will use the same notation for fully discrete problems as we have done in the semi-discrete setting, and simply treat time as another coordinate. In this case, we say that the fully discrete scheme is stable if, under the conditions of Definition 2.2.1, the estimate (2.9) is satisfied at the final time. 



\section{The energy method}

In many cases, the question of well-posedness may be addressed by applying the energy method. We introduce the method below by means of an example. Subsequently, we will define SBP operators and show how the discrete energy method yields energy estimates that mimic those of the continuous PDE.

\subsection{Strong boundary conditions}

Consider the following special case of (2.1):

$$
\begin{aligned}
u_{t}+u_{x}=0, & t>0, \quad x \in(0,1), \\
u=g, & t \geq 0, \quad x=0, \\
u=f, & t=0, \quad x \in[0,1] .
\end{aligned}
$$

Note that the boundary condition is defined only at the boundary point $x=$ 0 . We refer to formulations such as (3.1) as an IBVP with strong boundary conditions. As a special case of (2.2), we choose the inner product and norm

$$
(u, v)=\int_{0}^{1} u(x) v(x) \mathrm{d} x, \quad\|u\|=(u, u)^{1 / 2} .
$$

The energy method applied to (3.1) (multiplying the equation by the solution, integrating by parts and imposing the boundary condition) yields

$$
\frac{\mathrm{d}}{\mathrm{d} t}\|u(x, t)\|^{2}=g^{2}(t)-u(1, t)^{2} \leq g^{2}(t) .
$$

Comparing (3.2) with (2.3), we clearly have semi-boundedness with $\alpha=0$. Further, we cannot retain semi-boundedness if the boundary condition is removed, whence it is maximal. Temporal integration of (3.2) to time $t=T$ gives

$$
\|u(x, T)\|^{2} \leq\|f(x)\|^{2}+\int_{0}^{T} g^{2}(t) \mathrm{d} t .
$$

Comparing (3.3) with (2.6) from Definition 2.1.2, we see that (3.1) is strongly well-posed.

Herein lies the strength of the energy method; the procedure results in an evolution equation for the norm of the solution (the 'energy'). Integrating by parts 
produces a set of boundary terms. If these have the proper sign, a semi-bounded operator is obtained and an energy estimate is readily available. However, if they are of the wrong sign, there is in general no chance of bounding them in terms of $\|u\|$, and semi-boundedness is thus beyond reach.

\subsection{Weak formulations}

As an alternative to the strong formulation (3.1), we may define the boundary condition on the entire domain and impose it weakly as follows:

$$
\begin{aligned}
& u_{t}+u_{x}=\mathcal{L}(u-g), \quad t>0, \quad x \in[0,1], \\
& u=f, \quad t=0, \quad x \in[0,1] .
\end{aligned}
$$

Here, $\mathcal{L}$ is a lifting operator [2], defined for smooth functions $\phi$ and $\psi$ through the relation

$$
\int_{0}^{1} \phi \mathcal{L}(\psi) \mathrm{d} x=\left.\phi \psi\right|_{x=0} .
$$

The energy method applied to (3.4) yields

$$
\frac{\mathrm{d}}{\mathrm{d} t}\|u(x, t)\|^{2}=g^{2}(t)-u(1, t)^{2}-(u(0, t)-g)^{2} \leq g^{2}(t),
$$

and maximal semi-boundedness follows in the same way as for the strong formulation. Integrating (3.5) in time reproduces the energy estimate (3.3); hence (3.4) is strongly well-posed. Note that we may impose the initial condition weakly in a similar fashion.

\subsection{Transmission}

So far, we have considered a single PDE and seen that the energy method allows us to obtain an energy estimate, if appropriate boundary conditions are available. In other situations, we may have to solve two or more PDEs that are coupled across an interface. In this case, the energy method yields a set of interface terms that must be bounded. Coupling PDEs can be done both in space and in time. In the latter case, we refer to the resulting model as a transmission problem.

As a simple example, consider

$$
\begin{aligned}
& u_{t}+u_{x}=0, \quad 0<t<1, \quad x \in(0,1), \\
& v_{t}+v_{x}=0, \quad 1<t<2, \quad x \in(0,1) \text {, } \\
& u=0, \quad 0 \leq t \leq 1, \quad x=0, \\
& v=0, \quad 1 \leq t \leq 2, \quad x=0, \\
& u=f, \quad t=0, \quad x \in[0,1], \\
& v=u, \quad t=1, \quad x \in[0,1] \text {. }
\end{aligned}
$$


We seek an energy estimate for $v(x, 2)$ in terms of the initial data $f$. The energy method followed by integration in time results in

$$
\begin{aligned}
& \|u(x, 1)\|^{2} \leq\|f(x)\|^{2}, \\
& \|v(x, 2)\|^{2} \leq\|u(x, 1)\|^{2} .
\end{aligned}
$$

Adding the two equations immediately gives $\|v(x, 2)\|^{2} \leq\|f(x)\|^{2}$, and an energy estimate is obtained.

A more realistic problem arises when we wish to couple two problems of the general form (2.1) as follows:

$$
\begin{aligned}
& u_{t}+\mathcal{D}_{1}(u)=F_{1}, \quad t_{1}<t<t_{2}, \quad x \in \Omega, \\
& v_{t}+\mathcal{D}_{2}(v)=F_{2}, \quad t_{2}<t<t_{3}, \quad x \in \Omega \text {, } \\
& \mathcal{B}_{1}(u)=g_{1}, \quad t_{1} \leq t \leq t_{2}, \quad x \in \Gamma, \\
& \mathcal{B}_{2}(v)=g_{2}, \quad t_{2} \leq t \leq t_{3}, \quad x \in \Gamma, \\
& u=f_{1}, \quad t=t_{1}, \quad x \in \Omega \cup \Gamma, \\
& v=X u, \quad t=t_{2}, \quad x \in \Omega \cup \Gamma \text {. }
\end{aligned}
$$

Here, we have introduced the coupling matrix $X=X(x, t, u)$. We assume that in the decoupled situation, $X u=f_{2}$ where $f_{2}$ is data, there exist matrices $H_{1}$ and $H_{2}$ inducing norms akin to (2.2) such that $\left\|u\left(x, t_{2}\right)\right\|_{H_{1}}^{2}$ and $\left\|v\left(x, t_{3}\right)\right\|_{H_{2}}^{2}$ can be estimated in terms of $\left\|f_{1}\right\|_{H_{1}}^{2}$ and $\left\|f_{2}\right\|_{H_{2}}^{2}$ respectively.

It is in general non-trivial to obtain an energy estimate for $v\left(x, t_{3}\right)$ in the transmission problem (3.7). The topic of Paper $\mathrm{V}$ is the derivation of conditions on $H_{1}, H_{2}$ and $X$ such that this can be achieved. It turns out that the precise condition for the availability of an energy estimate is the same for continuous problems with strong or weak boundary conditions, semi-discrete problems and fully discrete problems using summation-by-parts in time (see Chapter 4). An energy estimate can be obtained if and only if

$$
H_{1}-X^{\top} H_{2} X \geq 0
$$

is satisfied at the transmission time $t=t_{2}$, where the inequality denotes positive semi-definiteness.

Evidently, (3.7) is a very general model, and it has a large number of important problems as special cases. Likewise, the number of possible approximation methods that fit the framework is very large. Therefore, (3.8) serves as a general condition with a wide range of applications. 



\section{Summation-by-parts}

The SBP-SAT framework is designed to mimic the energy rates obtained for continuous IBVPs. Thus, if the governing equations are well-posed, the corresponding discretisation will be stable. In this chapter we introduce the SBP-SAT procedure for semi-discrete and fully discrete problems by discretising the model problem (3.1).

\subsection{Semi-discretisations}

An SBP operator may be defined as follows:

Definition 4.1.1. A matrix $D=P^{-1} Q$ is an $S B P$ operator of order $q$ if

1. $D \mathbf{x}^{m}=m \mathbf{x}^{m-1}, \quad m=0, \ldots, q$,

2. $P=P^{T}>0$,

3. $Q+Q^{\top}=B=\operatorname{diag}(-1,0, \ldots, 0,1)$.

Thus, for a function $u(x, t), D \mathbf{u}$ approximates the spatial derivative $u_{x}$ on the grid $\mathbf{x}$. Here, $\mathbf{x}^{m}$ should be interpreted as elementwise exponentiation. We remark that there are generalisations of SBP operators [5] which will be discussed in due course.

Recall from (2.8) that our discrete inner product and norm take the form

$$
(\mathbf{u}, \mathbf{v})_{h}=\mathbf{u}^{\top} P \mathbf{v}, \quad\|\mathbf{u}\|_{h}=(\mathbf{u}, \mathbf{u})_{h}^{1 / 2} .
$$

Let us now make a comparison with the continuous setting. Consider the continuous inner product $\left(u, v_{x}\right)$ and integrate by parts:

$$
\left(u, v_{x}\right)=\int_{0}^{1} u v_{x} \mathrm{~d} x=\left.u v\right|_{0} ^{1}-\int_{0}^{1} u_{x} v \mathrm{~d} x=\left.u v\right|_{0} ^{1}-\left(u_{x}, v\right) .
$$

Analogously, using SBP operators, consider the discrete inner product

$$
(\mathbf{u}, D \mathbf{v})_{h}=\mathbf{u}^{\top} Q \mathbf{v}=\mathbf{u}^{\top}\left(B-Q^{\top}\right) \mathbf{v}=u_{n} v_{n}-u_{0} v_{0}-(D \mathbf{u}, \mathbf{v})_{h} .
$$


Thus, the SBP property ensures that the discretisation reproduces integration by parts. This is the key property that will allow us to discretely mimic continuous energy estimates.

A semi-discretisation of (3.1) is given by

$$
\mathbf{u}_{t}+P^{-1} Q \mathbf{u}=\sigma_{0} P^{-1}\left(u_{0}-g\right) \mathbf{e}_{0},
$$

where the right-hand side consists of an SAT term that weakly imposes the boundary condition at the first grid point. Thus, the SAT term is an example of a discrete lifting operator. Here, $\mathbf{e}_{0}$ is the first column of the identity matrix, and $\sigma_{0}$ is a penalty parameter that will be chosen to obtain semi-boundedness. The discrete energy method (multiplying by $\mathbf{u}^{T} P$, adding the transpose of the result and using Definition 4.1.1) applied to (4.1) yields

$$
\frac{\mathrm{d}}{\mathrm{d} t}\|\mathbf{u}\|_{h}^{2}=u_{0}^{2}\left(2 \sigma_{0}+1\right)-u_{n}^{2}-2 \sigma_{0} u_{0} g
$$

Choosing $\sigma_{0}=-1$ gives, after adding and subtracting $g^{2}$ on the right-hand side of $(4.2)$,

$$
\frac{\mathrm{d}}{\mathrm{d} t}\|\mathbf{u}\|_{h}^{2}=g^{2}-u_{n}^{2}-\left(u_{0}-g\right)^{2} \leq g^{2}
$$

which term for term mimics the continuous energy rate (3.5). As in the continuous case, maximal semi-boundedness is thus obtained. Integrating (4.3) in time gives

$$
\|\mathbf{u}(T)\|_{h}^{2} \leq\|\mathbf{f}\|_{h}^{2}+\int_{0}^{T} g^{2}(t) \mathrm{d} t .
$$

Comparing (4.4) with (2.10) in Definition 2.2.1, it is clear that the discretisation (4.1) is strongly stable.

\section{2 $\mathrm{SBP}$ in time}

It is possible to utilise the SBP-SAT framework for temporal as well as spatial discretisations [14]. The definition of an SBP operator acting in time is completely analogous to Definition 4.1.1. Before proceeding, we define the Kronecker product of two matrices $A$ and $B$ as

$$
A \otimes B=\left(\begin{array}{ccc}
a_{11} B & \ldots & a_{1 n} B \\
\vdots & \ddots & \vdots \\
a_{m 1} B & \ldots & a_{m n} B
\end{array}\right)
$$

The Kronecker product satisfies several interesting properties. Among them, we will need to use the facts that $(A \otimes B)^{\top}=\left(A^{\top} \otimes B^{\top}\right),(A \otimes B)^{-1}=\left(A^{-1} \otimes B^{-1}\right)$ and $(A \otimes B)(C \otimes D)=(A C \otimes B D)$, assuming that the matrices $C$ and $D$ have appropriate dimensions. 
A full discretisation of (3.1) is given by

$$
\begin{aligned}
\left(P_{t}^{-1} Q_{t} \otimes I_{x}\right) \mathbf{u}+\left(I_{t} \otimes P_{x}^{-1} Q_{x}\right) \mathbf{u} & =\left(\Sigma_{t} \otimes P_{x}^{-1}\right)\left(\left(\mathbf{u}_{x_{0}}-\mathbf{g}\right) \otimes \mathbf{e}_{x}\right) \\
& +\left(P_{t}^{-1} \otimes \Sigma_{x}\right)\left(\mathbf{e}_{t} \otimes\left(\mathbf{u}_{t_{0}}-\mathbf{f}\right)\right),
\end{aligned}
$$

where the subscripts $t, x$ indicate that the matrix dimension corresponds to the temporal and spatial grid respectively. Here, $\mathbf{e}_{x, t}$ denote the first column of the identity matrices $I_{t, x}$, and $\Sigma_{t, x}$ are penalty matrices yet to be determined. The elements of the vector $\mathbf{u}_{x_{0}}$ contain the numerical solution at the first spatial grid point projected onto the temporal grid, and similarly for $\mathbf{u}_{t_{0}}$. Finally, $\mathbf{f}$ and $\mathbf{g}$ contain the discrete initial and boundary data.

The energy method applied to (4.5) (multiplying by $\mathbf{u}^{\top}\left(P_{t} \otimes P_{x}\right)$, adding the transpose and using the properties of the SBP operators) yields, after some rearrangement,

$$
\begin{aligned}
\mathbf{u}_{t_{n}}^{\top} P_{x} \mathbf{u}_{t_{n}} & =\mathbf{u}_{t_{0}}^{\top} P_{x} \mathbf{u}_{t_{0}}+\mathbf{u}_{x_{0}}^{\top} P_{t} \mathbf{u}_{x_{0}}-\mathbf{u}_{x_{n}}^{\top} P_{t} \mathbf{u}_{x_{n}} \\
& +\mathbf{u}_{x_{0}}^{\top} P_{t} \Sigma_{t}\left(\mathbf{u}_{x_{0}}-\mathbf{g}\right)+\left(\mathbf{u}_{x_{0}}-\mathbf{g}\right)^{\top} \Sigma_{t}^{\top} P_{t} \mathbf{u}_{x_{0}} \\
& +\mathbf{u}_{t_{0}}^{\top} P_{x} \Sigma_{x}\left(\mathbf{u}_{t_{0}}-\mathbf{f}\right)+\left(\mathbf{u}_{t_{0}}-\mathbf{f}\right)^{\top} \Sigma_{x}^{\top} P_{x} \mathbf{u}_{t_{0}}
\end{aligned}
$$

where $\mathbf{u}_{t_{n}}$ and $\mathbf{u}_{x_{n}}$ denote the solution vector at the final temporal and spatial grid point respectively (although the temporal and spatial grids of course may have different dimensions). Choosing $\Sigma_{t}=-I_{t}$ and $\Sigma_{x}=-I_{x}$, then adding and subtracting $\mathbf{f}^{\top} P_{x} \mathbf{f}$ and $\mathbf{g}^{\top} P_{t} \mathbf{g}$ to (4.6) results in

$$
\begin{aligned}
\mathbf{u}_{t_{n}}^{\top} P_{x} \mathbf{u}_{t_{n}} & =\mathbf{f}^{\top} P_{x} \mathbf{f}-\left(\mathbf{u}_{\mathbf{t}_{\mathbf{o}}}-\mathbf{f}\right)^{\top} P_{x}\left(\mathbf{u}_{\mathbf{t}_{\mathbf{o}}}-\mathbf{f}\right) \\
& +\mathbf{g}^{\top} P_{t} \mathbf{g}-\left(\mathbf{u}_{\mathbf{x}_{\mathbf{0}}}-\mathbf{g}\right)^{\top} P_{t}\left(\mathbf{u}_{\mathbf{x}_{\mathbf{0}}}-\mathbf{g}\right)-\mathbf{u}_{x_{n}}^{\top} P_{t} \mathbf{u}_{x_{n}} \\
& \leq \mathbf{f}^{\top} P_{x} \mathbf{f}+\mathbf{g}^{\top} P_{t} \mathbf{g} .
\end{aligned}
$$

The energy estimate (4.7) is term for term analogous to the continuous estimate (3.3). Note that the discretisation is slightly dissipative since $\mathbf{x}_{\mathbf{0}}-\mathbf{g}$ and $\mathbf{u}_{t_{0}}-\mathbf{f}$ may be non-zero. This effect vanishes with grid refinement. Clearly the full discretisation (4.5) is strongly stable.

\subsection{Second derivatives}

The SBP framework may be extended to second (and higher) derivatives [10]. We have reason to do so in the numerical experiments in Paper III. To obtain the appropriate symmetry conditions for a second derivative SBP operator, we consider the diffusion equation, $u_{t}=u_{x x}$. The energy method results in

$$
\frac{\mathrm{d}}{\mathrm{d} t}\|u\|^{2}=\left.2 u u_{x}\right|_{0} ^{1}-2\left\|u_{x}\right\|^{2},
$$

where we have used the same inner product and norm as before. We wish to mimic this energy rate using a second order SBP operator, $D_{2}$. A suitable ansatz is $D_{2}=P^{-1}\left(-D_{1}^{\top} P D_{1}+B S\right)$, where $D_{1}$ is a consistent first derivative 
approximation, $S$ is an approximation of the first derivative at the boundaries, and $B$ is given in Definition 4.1.1. Semi-discretising gives $\mathbf{u}_{t}=D_{2} \mathbf{u}$, and the energy method results in

$$
\frac{\mathrm{d}}{\mathrm{d} t}\|\mathbf{u}\|_{h}^{2}=2 u_{n}(S \mathbf{u})_{n}-2 u_{0}(S \mathbf{u})_{0}-2\left\|D_{1} u\right\|_{h}^{2} .
$$

Clearly (4.9) mimics the continuous energy rate (4.8).

Letting $D_{2}=D^{2}$, where $D$ is a first derivative SBP operator in the sense of Definition 4.1.1, yields a second derivative operator that satisfies the ansatz. To see this, note that

$$
D_{2}=P^{-1}(Q D)=P^{-1}\left(\left(B-Q^{\top}\right) D\right)=P^{-1}\left(-D^{\top} P D+B D\right) .
$$

There are other ways of designing $D_{2}$ that enjoys certain advantages compared to using the first derivative twice, however the above approach suffices for our needs.

\subsection{Generalised SBP}

Consider an IBVP posed on a spatial domain $x \in[a, b]$. Generalised SBP (gSBP) operators [5] are obtained by relaxing the property $Q+Q^{\top}=B$ as follows:

Definition 4.4.1. A matrix $D=P^{-1} Q$ is a $g S B P$ operator of order $q$ if

1. $D \mathbf{x}^{m}=m \mathbf{x}^{m-1}, \quad m=0, \ldots, q$,

2. $P=P^{T}>0$,

3. $Q+Q^{T}=e_{b}^{T} e_{b}-e_{a}^{T} e_{a}$,

4. $e_{a} \mathbf{x}^{m}=a^{m}, \quad e_{b} \mathbf{x}^{m}=b^{m} \quad m=0, \ldots, q$,

that $i s, e_{a, b}$ are boundary interpolation operators defined on the grid $\mathbf{x}$.

Such operators enjoy the freedom of utilising grids that do not necessarily conform with the physical domain boundaries. In case they do conform with the boundaries, Definition 4.4.1 and 4.1.1 are equivalent. We shall return to gSBP operators in Paper IV as well as in the next chapter, though under the less precise moniker 'SBP operators'. 


\section{Finite difference operators}

The definition of an SBP operator specifies certain symmetry conditions on the matrices $P$ and $Q$. This is sufficient to obtain energy estimates, as we have shown in the previous chapter. However, up to this point we have not given these matrices any further meaning. In the context of finite element methods, $P$ and $Q$ may respectively be identified as the mass and stiffness matrices, and their meanings are derived thence. For spectral methods, it is natural to think of $P$ as a Gaussian quadrature rule. In Papers I-IV we assume that the SBP operator constitutes a finite difference approximation. This chapter outlines the properties of $P$ and $Q$ in this specific context, with particular focus on structure and accuracy.

\subsection{The structure of $P$ and $Q$}

In this chapter, we consider a physical domain $x \in[a, b]$ and let the spatial grid be given by

$$
\mathbf{x}=\left(x_{0}, x_{1}, \ldots, x_{r}, x_{r}+h, x_{r}+2 h, \ldots\right)^{T},
$$

where $x_{0}<x_{1}<\cdots<x_{r}$ and $h$ is the grid spacing in the interior of the domain. In papers I-III it is assumed that the grid is uniform, i.e. that $x_{j}=$ $x_{0}+j h$ for all $j$, and further that $x_{0}=a$, i.e. it coincides with the physical domain boundary. In paper IV these conditions are relaxed, and in paper $\mathrm{V}$, the structure of the grid is completely arbitrary. Here, we will let $x_{0}, \ldots, x_{r}$ be arbitrarily distributed, and resort to Definition 4.4.1 for the generalised SBP operators.

We let an SBP operator be defined on the grid $\mathbf{x}$ in (5.1), and further let it be based on finite difference stencils. By this we mean that $Q$ is a banded matrix which, away from boundaries, assumes the form of a repeated interior stencil consisting of central finite differences. In order to close the operator near boundaries, the stencils are modified and become progressively more one-sided. 
The most general form of $Q$ near the left boundary is

$$
Q=\left(\begin{array}{cccccccccc}
q_{0,0} & \ldots & q_{0, r} & \ldots & q_{0, r+n-1} & & & & & \\
\vdots & \ddots & \vdots & & \vdots & & & & \\
q_{r, 0} & \ldots & q_{r, r} & \ldots & q_{r, r+n-1} & a_{n} & & & \\
\vdots & & \vdots & \ddots & \vdots & \vdots & \ddots & & & \\
q_{r+n-1,0} & \ldots & q_{r+n-1, r} & \ldots & q_{r+n-1, r+n-1} & a_{1} & \ldots & a_{n} & & \\
& & -a_{n} & \ldots & -a_{1} & 0 & a_{1} & \ldots & a_{n} & \\
& & & \ddots & & \ddots & \ddots & \ddots & & \ddots
\end{array}\right) .
$$

Here, $q_{i j}$ are the stencil coefficients of the boundary blocks. The parameters $a_{1}, \ldots, a_{n}$ are the coefficients of the repeated central difference stencil, defined to be of order $2 s, s>0$, through the conditions

$$
\frac{\partial u}{\partial x}=\frac{1}{h} \sum_{k=1}^{n} a_{k}(u(x+k h, t)-u(x-k h, t))+\mathcal{O}\left(h^{2 s}\right) .
$$

Note that the interior stencil by definition operates on a uniform grid. However, recall from (5.1) that $r$ determines the number of non-uniformly distributed grid points. Hence, the central difference stencil may not utilise any of the first $r$ grid points, which is why $n-1$ extra rows are required in the boundary block to close the SBP operator. Note that if $r=0$ we recover a uniform grid.

We let the matrix $P$ take the form of a diagonal matrix,

$$
P=h \operatorname{diag}\left(p_{0}, \ldots, p_{r+n-1}, 1,1, \ldots\right) .
$$

From Definition 4.4.1 it is clear that in order to ensure positive definiteness, we must have $p_{j}>0, j=0, \ldots, r+n-1$. Note that the diagonal elements of $P$ are equal to $h$ everywhere except in the $r+n$ rows corresponding to the boundary block of $Q$.

The definition of the SBP operator does not specify that $P$ must be diagonal, and indeed it is possible to construct operators that use other structures $[9$, 16]. However, the stability of such operators may deteriorate for problems with variable coefficients [11]; hence we will not deliberate them further.

Throughout this thesis we assume that the right boundary is treated in the same way as the left one. In practice, this means that $p_{N-j}=p_{j}$ and $q_{N-i, N-j}=$ $-q_{i, j}$. This assumption implies that the grid points are placed symmetrically with respect to the physical domain, i.e. the distance from the left boundary $a$ to $x_{j}$ is the same as the distance from $x_{N-j}$ to the right boundary $b$.

\subsection{Accuracy and convergence}

In general, an SBP operator $D$ is less accurate near the boundary than in the interior. We let $q$ be the order of accuracy of the boundary block, and $2 s$ the 
order of the interior stencil. Then, the operator differentiates polynomials up to order $q$ exactly, as indicated by Definitions 4.1.1 and 4.4.1. For a smooth function $f(x)$, this further implies that the error satisfies

$$
D \mathbf{f}-\mathbf{f}^{\prime}=\mathcal{O}\left(h^{q}\right),
$$

as is verified by a Taylor expansion. Here, $\mathbf{f}$ and $\mathbf{f}^{\prime}$ are projections of $f$ and its derivative onto the grid (5.1). A fundamental result states that the convergence rate of an SBP discretisation is $q+1$ for first order hyperbolic problems, and $q+2$ for first order parabolic or second order hyperbolic problems, if the discretisation is pointwise stable [17]. Naturally, it is desirable to have $q \geq 2 s-1$ in order to preserve the convergence rate of the interior stencil. A classic result for uniform, conforming grids dictates that this is impossible when $P$ is a diagonal matrix. In fact, it is in general impossible to choose $q>s$ [9]. A natural question is whether it is possible to remedy this drawback by departing from uniformity and conformity, and defining the operator on the arbitrary grid (5.1). Such strategies are commonly used to increase the order of accuracy of spectral methods. A major result of Paper IV answers this question in the negative; it is not possible to choose $q>s$ on any grid. In practice, the choice $q=s$ is essentially always made.

Another important result derived in Paper IV is that if $q=s$, then $P$ defines a quadrature rule of order $2 q$. This has previously been established on uniform grids [7]. A first indication to why this assertion is true is given by the so called compatibility conditions [9]: Recall from Definition 4.4.1 that $D \mathbf{x}^{j}=j \mathbf{x}^{j-1}, j=$ $0, \ldots q$. We multiply from the left by $\left(\mathbf{x}^{i}\right)^{T} P$ to obtain

$$
\left(\mathbf{x}^{i}\right)^{T} Q \mathbf{x}^{j}=j\left(\mathbf{x}^{i}\right)^{T} P \mathbf{x}^{j-1}=j \mathbf{1}^{T} P \mathbf{x}^{i+j-1},
$$

where in the last equality we have used the facts that $P$ is diagonal and that $\mathbf{x}^{0}=\mathbf{1}$, i.e. the grid vector of all ones. Now swap the indices $i$ and $j$ and add the result to obtain the simplest form of the compatibility conditions;

$$
\left(\mathbf{x}^{i}\right)^{T}\left(Q+Q^{T}\right) \mathbf{x}^{j}=(i+j) \mathbf{1}^{T} P \mathbf{x}^{i+j-1}, \quad i, j=0, \ldots, q .
$$

From the properties of $Q$ specified in Definition 4.4.1, it follows that

$$
(i+j) \mathbf{1}^{T} P \mathbf{x}^{i+j-1}=b^{i+j}-a^{i+j}, \quad i, j=0, \ldots, q .
$$

In other words, $P$ integrates polynomials up to order $2 q-1$ exactly. It can be shown [1] that for a smooth function $f(x)$, this implies that the error

$$
\left|\int_{a}^{b} f(x)-\mathbf{1}^{T} P \mathbf{f}\right|=\mathcal{O}\left(h^{2 q}\right),
$$

whence $P$ does indeed define a quadrature rule of order $2 q$ as asserted. In Paper IV it is further shown that this order cannot be exceeded. 



\section{Dispersion}

A fundamental property of PDEs describing wave motion is the dispersion relation, which encodes properties such as the wave speed into the model. Discretisations have their own numeric dispersion relations, and in general they are different from the analytic one. This chapter briefly introduces the theory of dispersion errors of finite difference stencils and discusses their implications and remedies. We shall concern ourselves exclusively with spatial discretisations, and thus leave the time derivatives intact. For simplicity of presentation, we restrict our attention to central difference stencils. Complications arising at domain boundaries and interfaces are considered in Paper III.

\subsection{Analytic and numeric dispersion relations}

Let us return to problem (3.1) but change the setting to a periodic domain. We ignore initial conditions such that we may concern ourselves exclusively with the PDE;

$$
u_{t}+u_{x}=0, \quad 0 \leq t .
$$

Suppose that $u(x, t)$ has a uniformly convergent Fourier expansion. We may then consider solutions of (6.1) taking the form of a general Fourier mode, $u(x, t)=\exp (i(\kappa x-\omega t))$, with wavenumber $\kappa$ and angular frequency $\omega$. Inserting $u$ into (6.1) and solving for $\omega$ gives

$$
\omega=\kappa .
$$

The phase speed $v_{p}$, and group speed $v_{g}$, are respectively defined as

$$
v_{p}:=\frac{\omega}{\kappa}=1, \quad v_{g}:=\frac{\partial \omega}{\partial \kappa}=1 .
$$

Thus (6.1) describes a wave travelling to the right at unit speed. The relation (6.2) is known as the analytic dispersion relation. The problem (6.1) is nondispersive in the sense that the phase speed is independent of $\omega$ and $\kappa$, i.e. all waves of any frequency propagate equally fast.

Recall from (5.2) that a central difference stencil of order $2 s$ is given by

$$
\left.\frac{\partial u}{\partial x}\right|_{x_{j}} \approx D_{1} u_{j}:=\frac{1}{h} \sum_{k=1}^{n} a_{k}\left(u_{j+k}(t)-u_{j-k}(t)\right) .
$$


Discretising (6.1) in space thus gives

$$
\frac{\mathrm{d} u_{j}}{\mathrm{~d} t}+D_{1} u_{j}=0
$$

Again, inserting a general Fourier mode $u_{j}(t)=\exp \left(i\left(\kappa x_{j}-\bar{\omega} t\right)\right)$ and solving for the frequency $\bar{\omega}$ gives the numeric dispersion relation

$$
\bar{\omega}=\frac{2}{h} \sum_{k=1}^{n} a_{k} \sin (k h \kappa) .
$$

The corresponding phase and group speeds are

$$
\bar{v}_{p}:=\frac{\bar{\omega}}{\kappa}, \quad \bar{v}_{g}:=\frac{\partial \bar{\omega}}{\partial \kappa} .
$$

Note from (6.3) and (6.4) that $\bar{v}_{p}$ and $\bar{v}_{g}$ depend on the wavenumber, $\kappa$. The approximation is thus inherently dispersive, which is what gives rise to dispersion errors and subsequent errors in phase and group velocity.

It is convenient to introduce the normalised wavenumber $\xi=\kappa h$ and similarly normalise (6.3) with $h$;

$$
\bar{\xi}:=\bar{\kappa} h \equiv \bar{\omega} h=2 \sum_{k=1}^{n} a_{k} \sin (k \xi) .
$$

Here we have simply defined $\bar{\kappa}=\bar{\omega}$ in analogy with (6.2). Noting that the smallest wavelength the scheme can resolve is $\lambda_{\min }=2 h$, we find that the largest resolvable wavenumber is $\kappa_{\max }=2 \pi / \lambda_{\min }=\pi / h$. Thus we always have $|\xi| \leq \pi$.

The dispersion error is defined as the discrepancy between the analytic and numeric wavenumbers;

$$
E_{d}(\xi):=\xi-\bar{\xi}
$$

Generally, $E_{d}$ scales as $\mathcal{O}\left(h^{2 s+1}\right)$ for a central difference stencil of order $2 s$, and sufficiently small $h$. In Figure $6.1, \bar{\xi}$ is plotted as a function of $\xi$ for some common central difference stencils. Clearly, small wavenumbers are better approximated than large ones, and high order methods are better than low order ones. High order methods can thus be used on coarser meshes than their low order counterparts, which is beneficial from an efficiency point of view. The topic of Papers I-II is the derivation and implementation of new, optimised finite difference stencils with even smaller dispersion error than the highest order methods, for a given number of stencil coefficients. Extensions to SBP operators are presented in Paper III.

\subsection{Filters}

We generally say that a Fourier mode of frequency $\omega$ is resolved if the corresponding dispersion error $E_{d}(\xi)$ is sufficiently small. Figure 6.1 clearly demonstrates that higher order methods have better resolution capacity than lower 


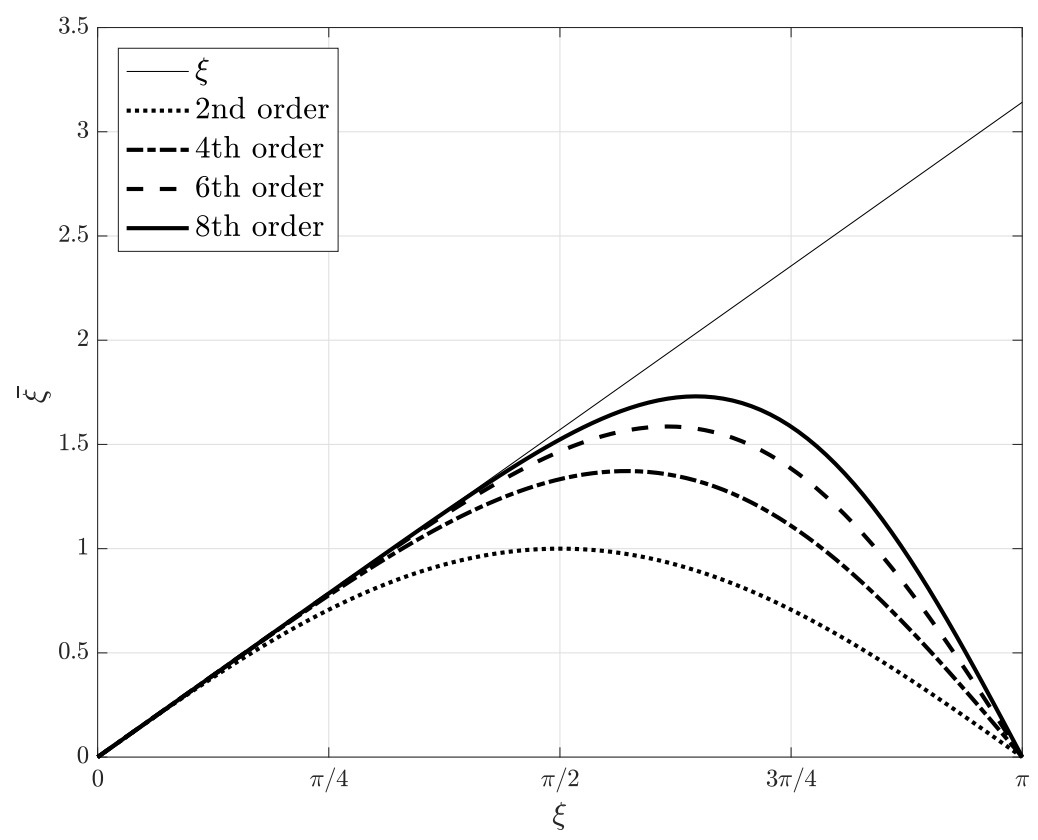

Figure 6.1: Numerical wavenumbers associated with central difference stencils.

order ones. Nevertheless, no finite difference stencil has satisfactory resolution in the vicinity of $\xi=\pi$, which is the source of aliasing errors, causing instabilities for non-linear problems. A candidate strategy to handle this issue is to effectively remove high-frequency content from the numerical solution. One way of achieving this is by introducing a numerical filter. Filters are discussed both in Paper II and Paper V in different contexts. We briefly introduce their properties below.

The idea of a filter is to operate on the solution in such a way that its energy content is biased towards the resolved wavenumbers. The solution $u_{j}$ can be expressed as a sum of Fourier modes of the general form $u_{j}=\sum_{\nu} A_{\nu} \cos \nu\left(x_{j}-\phi_{\nu}\right)$, where $A_{\nu}(t)$ is the amplitude of the mode and $\phi_{\nu}$ is the phase. We seek an amplitude response function, $R(\xi)$, such that the filter renormalises the amplitudes of the Fourier modes as $R A_{\nu}$.

A useful response function must satisfy several criteria: It should damp or completely remove the unresolved high-frequency Fourier modes while leaving the well resolved low-frequency spectrum virtually untouched. It should have a slope that approaches zero as $\xi \rightarrow 0$ with order $2 s$ such that the accuracy of the stencil is not compromised. It should be everywhere positive and its magnitude should be less than unity in order to prevent the growth of any 
Fourier component. A simple function that satisfies these requirements is

$$
R(\xi)=1-\sin ^{2 s} \frac{\xi}{2}
$$

although there are other options.

A candidate approach to designing an explicit filter is to introduce an approximation of the $2 s$ th derivative operator;

$$
\left.\frac{\partial^{2 s} u}{\partial x^{2 s}}\right|_{x_{j}} \approx D_{2 s} u_{j}:=\frac{1}{h^{2 s}}\left[b_{0} u_{j}+\sum_{k=1}^{n} b_{k}\left(u_{j+k}+u_{j-k}\right)\right] .
$$

Inserting the Fourier mode $u_{j}=\exp \left(i \kappa x_{j}\right)$ in the right-hand side of $(6.8)$ gives the Fourier image

$$
\psi(\xi):=b_{0}+2 \sum_{k=1}^{n} b_{k} \cos (k \xi)
$$

If the approximation is second order accurate and $n=s$ in (6.8), it can be shown [8] that

$$
\psi(\xi)=(-1)^{s+1}\left(2 \sin \frac{\xi}{2}\right)^{2 s}
$$

Thus, letting $F=\left(1+\alpha D_{2 s}\right)$, where $\alpha=(-1)^{s} 2^{-2 s}$, results in a filter $F$ with response function (6.7).

While we will consider filters derived as above in Paper V, we emphasise that this is one among many possible designs. In Paper II, we utilise an optimised filter, chosen to match the properties of an optimised central difference stencils derived in Paper I. The resulting scheme has a resolution capacity akin to very high order methods while retaining the efficiency of a relatively narrow difference stencil. 


\section{Summary of papers}

\section{Paper I: Uniformly best wavenumber approxima- tions by spatial central difference operators}

Consider the central finite difference stencil (5.2). From (6.5) we know that its associated dispersion error is given by

$$
\xi-2 \sum_{k=1}^{n} a_{k} \sin (k \xi) .
$$

In Paper I we study the properties of the dispersion error associated with classical central differences, i.e. those with maximal order of accuracy for a given bandwidth. It is shown that the error is monotonically increasing with respect to $\xi$, and consequently that the numerical wavespeed necessarily underestimates the true wavespeed associated with the governing equation.

A strategy for devising new stencils with improved dispersion properties is developed. Rather than maximising the order of accuracy with respect to the bandwidth, a set of stencil parameters are used to minimise the dispersion error in the $L^{\infty}$-sense. It is shown that for each order and bandwidth, a unique optimal stencil exists that may be characterised in terms of the extrema of its dispersion error. New stencils are derived and compared with classical stencils as well as other low-dispersion methods, showing favourable results for a linear and a non-linear test case.

\section{Paper II: A simple and efficient incompressible Navier-Stokes solver for unsteady complex geom- etry flows on truncated domains}

In Paper II, the stencils from Paper I are tested in a realistic setting. The entropically damped artificial compressibility formulation of the incompressible Navier-Stokes equations is solved using the optimised stencils in combination with an optimised filter of the form (6.8). This, in turn, is combined with an immersed boundary method to efficiently treat complex geometries, and a new robust outflow boundary condition to enable higher Reynolds number simulations on truncated domains. Validation studies for the Taylor-Green vortex 
problem and the lid driven cavity problem in both $2 \mathrm{D}$ and $3 \mathrm{D}$ are presented. An eddy viscosity subgrid-scale model is used to enable large eddy simulations for the 3D cases. The resulting scheme is shown to be able to capture strong velocity gradients on relatively coarse grids compared to previously published studies.

\section{Paper III: Summation-by-parts operators with min- imal dispersion error for coarse grid flow calcula- tions}

In Paper III, SBP operators with minimal dispersion errors are constructed in a way analogous to the central difference stencils from Paper I and II. Their performance is investigated for severely under-resolved problems in combination with small amounts of artificial dissipation. It is shown that performance benefits can be obtained with appropriately optimised operators for wave propagation and turbulent flows involving large wavenumbers, long simulation times and large ranges of resolution scales. Further, the optimised operators remain stable for non-linear problems with less artificial dissipation than high order, non-optimal operators.

\section{Paper IV: On the order of accuracy of finite differ- ence operators on diagonal norm based summation- by-parts form}

A finite difference SBP operator of order $q$ near the boundaries and order $2 s$ in the interior necessarily satisfies $q \leq s$, if uniform and conforming grids are used. Paper IV investigates the possibility of increasing $q$ by departing from uniformity and conformity, as is frequently done for spectral methods. It is proven that $q \leq s$ must hold for any grid, irrespective of the point distribution near the domain boundaries. Further, it is shown that if $q=s$, the SBP matrix $P$ defines a quadrature rule of order $2 q$ irrespective of the choice of grid, and that this order cannot be exceeded.

\section{Paper V: Well-posed and stable transmission prob- lems}

In Paper V, we study the transmission problem (3.7), which describes the temporal coupling of two or more IBVPs by enforcing a transmission condition, $v\left(x, t_{2}\right)=X u\left(x, t_{2}\right)$, at some time $t=t_{2}$. Here, $X$ is a completely general, possibly solution-dependent matrix, describing the information flow from the first IBVP to the second one.

The problem of obtaining energy estimates for transmission problems is investigated. Continuous problems with strong and weak boundary conditions, 
semi-discretisations and fully discrete problems using SBP in time are analysed. It is shown that an energy estimate can be obtained if and only if the condition

$$
H_{1}-X^{\top} H_{2} X \geq 0
$$

is satisfied at the transmission time $t=t_{2}$, where the matrices $H_{1,2}$ induce the norms in which $u$ and $v$ are estimated (see (2.2)). Further, it is shown that the condition (7.1) can always be satisfied if the matrices $H_{1,2}$ are appropriately scaled; however this has a non-negligible impact on the sharpness of the resulting energy estimate. The theory is applied to a range of realistic situations, including coupled fluid flows, multi-grid accelerators, interpolatory multi-block formulations and explicit numerical filters. 


\section{References}

[1] B. K. Alpert. Hybrid Gauss-trapezoidal quadrature rules. SIAM Journal on Scientific Computing, 20(5):1551-1584, 1999.

[2] D. N. Arnold, F. Brezzi, B. Cockburn, and L. D. Marini. Unified analysis of discontinuous galerkin methods for elliptic problems. SIAM journal on numerical analysis, 39(5):1749-1779, 2002.

[3] M. H. Carpenter and D. Gottlieb. Spectral methods on arbitrary grids. Journal of Computational Physics, 129:74-86, 1996.

[4] M. H. Carpenter, D. Gottlieb, and S. Abarbanel. Time-stable boundary conditions for finite-difference schemes solving hyperbolic systems: methodology and application to high-order compact schemes. Journal of Computational Physics, 111(2):220-236, 1994.

[5] D. C. D. R. Fernández, P. D Boom, and D. W. Zingg. A generalized framework for nodal first derivative summation-by-parts operators. Journal of Computational Physics, 266:214-239, 2014.

[6] G. J. Gassner. A skew-symmetric discontinuous Galerkin spectral element discretization and its relation to SBP-SAT finite difference methods. SIAM Journal on Scientific Computing, 35(3):A1233-A1253, 2013.

[7] J. E. Hicken and D. W. Zingg. Summation-by-Parts operators and highorder quadrature. Journal of Computational and Applied Mathematics, 237:111-125, 2013.

[8] C. A. Kennedy and M. H. Carpenter. Comparison of several numerical methods for simulation of compressible shear layers. In NASA technical paper 3438, Langley research, 1997.

[9] H.-O. Kreiss and G. Scherer. Finite element and finite difference methods for hyperbolic partial differential equations. Aspects of Finite Elements in Partial Differential Equations, Academic Press, Inc., 1974.

[10] K. Mattsson and J. Nordström. Summation-by-Parts operators for finite difference approximations of second derivatives. Journal of Computational Physics, 199(2):503-540, 2004.

[11] J. Nordström. Conservative finite difference formulations, variable coefficients, energy estimates and artificial dissipation. J. Sci. Comput., 29:375404, 2006.

[12] J. Nordström and M. Björck. Finite volume approximations and strict stability for hyperbolic problems. Applied numerical mathematics, 38(3):237$255,2001$. 
[13] J. Nordström, K. Forsberg, C. Adamsson, and P. Eliasson. Finite volume methods, unstructured meshes and strict stability for hyperbolic problems. Applied Numerical Mathematics, 45(4):453-473, 2003.

[14] J. Nordström and T. Lundquist. Summation-by-Parts in time. Journal of Computational Physics, 251:487-499, 2013.

[15] H. Ranocha, P. Öffner, and T. Sonar. Summation-by-Parts operators for correction procedure via reconstruction. Journal of Computational Physics, 311:299-328, 2016.

[16] B. Strand. Summation-by-Parts for finite difference approximations for d/dx. Journal of Computational Physics, 110:47-67, 1994.

[17] M. Svärd and J. Nordström. On the order of accuracy for difference approximations of initial-boundary value problems. Journal of Computational Physics, 218(1):333-352, 2006. 


\section{Papers}

The papers associated with this thesis have been removed for copyright reasons. For more details about these see:

http://urn.kb.se/resolve?urn=urn:nbn:se:liu:diva- 143059 